\title{
Characterizing and optimizing a laser-desorption molecular beam source
}

Nicole Teschmit, Karol Długołęcki, Daniel Gusa, Igor Rubinsky, Daniel A. Horke, and Jochen Küpper

Citation: The Journal of Chemical Physics 147, 144204 (2017); doi: 10.1063/1.4991639

View online: http://dx.doi.org/10.1063/1.4991639

View Table of Contents: http://aip.scitation.org/toc/jcp/147/14

Published by the American Institute of Physics

\section{Articles you may be interested in}

Formic acid aggregation in 2D supersonic expansions probed by FTIR imaging

The Journal of Chemical Physics 147, 144305 (2017); 10.1063/1.4989544

Coherent laser-millimeter-wave interactions en route to coherent population transfer

The Journal of Chemical Physics 147, 144201 (2017); 10.1063/1.4997624

Communication: General variational approach to nuclear-quadrupole coupling in rovibrational spectra of polyatomic molecules

The Journal of Chemical Physics 147, 141101 (2017); 10.1063/1.5002533

A multi-plate velocity-map imaging design for high-resolution photoelectron spectroscopy

The Journal of Chemical Physics 147, 094201 (2017); 10.1063/1.4996011

Extracting spectroscopic molecular parameters from short pulse photo-electron angular distributions

The Journal of Chemical Physics 147, 144304 (2017); 10.1063/1.4998818

Controlling the crystal polymorph by exploiting the time dependence of nucleation rates

The Journal of Chemical Physics 147, 144505 (2017); 10.1063/1.4993566

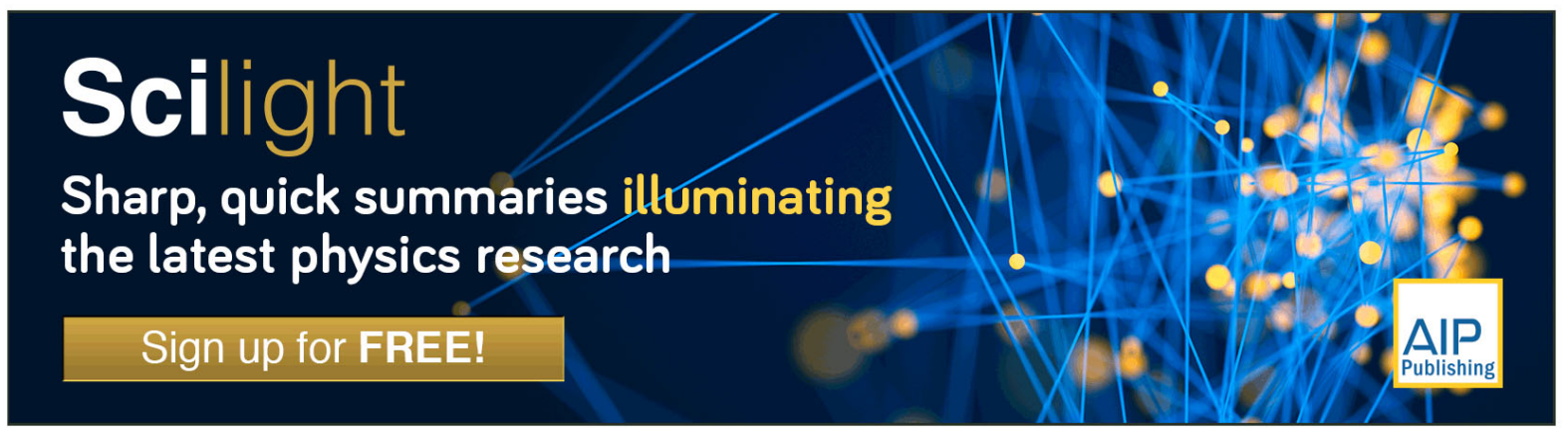




\title{
Characterizing and optimizing a laser-desorption molecular beam source
}

\author{
Nicole Teschmit, ${ }^{1,2,3}$ Karol Długołęcki, ${ }^{1}$ Daniel Gusa, ${ }^{1}$ Igor Rubinsky, ${ }^{1}$ Daniel A. Horke, ${ }^{1,2}$ \\ and Jochen Küpper ${ }^{1,2,3,4, a)}$ \\ ${ }^{1}$ Center for Free-Electron Laser Science, Deutsches Elektronen-Synchrotron DESY, Notkestrasse 85, \\ 22607 Hamburg, Germany \\ ${ }^{2}$ The Hamburg Center for Ultrafast Imaging, Universität Hamburg, Luruper Chaussee 149, \\ 22761 Hamburg, Germany \\ ${ }^{3}$ Department of Chemistry, Universität Hamburg, Martin-Luther-King-Platz 6, 20146 Hamburg, Germany \\ ${ }^{4}$ Department of Physics, Universität Hamburg, Luruper Chaussee 149, 22761 Hamburg, Germany
}

(Received 22 June 2017; accepted 20 September 2017; published online 13 October 2017)

\begin{abstract}
The design and characterization of a new laser-desorption molecular beam source, tailored for use in $\mathrm{x}$-ray free-electron laser and ultrashort-pulse laser imaging experiments, is presented. It consists of a single mechanical unit containing all source components, including the molecular-beam valve, the sample, and the fiber-coupled desorption laser, which is movable in five axes, as required for experiments at central facilities. Utilizing strong-field ionization, we characterize the produced molecular beam and evaluate the influence of desorption laser pulse energy, relative timing of valve opening and desorption laser, sample bar height, and which part of the molecular packet is probed on the sample properties. Strong-field ionization acts as a universal probe and allows detecting all species present in the molecular beam, and hence enables us to analyze the purity of the produced molecular beam, including molecular fragments. We present optimized experimental parameters for the production of the purest molecular beam, containing the highest yield of intact parent ions, which we find to be very sensitive to the placement of the desorbed-molecule plumes within the supersonic expansion. Published by AIP Publishing. https://doi.org/10.1063/1.4991639
\end{abstract}

\section{INTRODUCTION}

Laser desorption (LD) is a widely used technique to vaporize non-volatile organic molecules for gas-phase studies. The concept of LD is a rapid heating of the sample to be vaporized, at around $10^{10}-10^{12} \mathrm{~K} / \mathrm{s}$, such that a fraction of molecules desorb intact instead of fragmenting. ${ }^{1,2}$ Later studies combined LD with pulsed molecular beams to directly cool the desorbed molecules, enabling the investigation of intact neutral molecules in the gas-phase at low vibrational temperatures..$^{3-6}$ The main advantage of $\mathrm{LD}$ over other vaporization techniques, such as thermal vaporization, is the ability to introduce intact thermally labile organic molecules, including peptides and proteins, into a cold molecular beam. This has been demonstrated, e.g., for a pentapeptide (Ser-Ile-Val-Ser-Phe- $\left.\mathrm{NH}_{2}\right)^{7}$ or the delta sleep inducing nonapeptide. ${ }^{8}$

A first detailed characterization of a LD source coupled to a molecular beam (MB) valve was conducted nearly 30 years ago. Using anthracene, diphenylamine, and perylene combined with resonance-enhanced multiphoton ionization (REMPI) spectroscopy, approximate vibrational temperatures of $<15 \mathrm{~K}$ and rotational temperatures of $5-10 \mathrm{~K}$ were determined. ${ }^{4}$ This demonstrated the ability of LD-MB to gently vaporize large, thermally labile molecules and to efficiently cool them. Since then, various spectroscopic techniques have been combined with LDMB sources, and

a)Electronic mail: jochen.kuepper@cfel.de. URL: https://www.controlledmolecule-imaging.org. recent experiments have included resonance-enhanced multiphoton ionization studies, ${ }^{5}$ (far) infrared (IR)-ultraviolet (UV) double resonance techniques, ${ }^{9}$ IR multiphoton dissociation, ${ }^{10}$ and zero-kinetic-energy-photoelectron (ZEKE) spectroscopy. ${ }^{11}$

In recent years, $x$-ray free-electron lasers (XFELs) have emerged as powerful tools for the structure determination of gas-phase systems, with the potential to achieve atomicresolution structures with femtosecond temporal resolution, recording the so-called molecular movies. ${ }^{12}$ The ultrashort pulse duration available at XFELs enables the recording of a diffraction pattern from a molecule prior to destruction by the high intensity of the $\mathrm{x}$-ray pulses. ${ }^{13}$ This diffraction-beforedestruction paradigm, albeit still discussed, ${ }^{14-16}$ has recently also been demonstrated for isolated gas-phase molecules. ${ }^{17-19}$ Similar to the time-resolved nuclear dynamics that can be recorded at XFEL sources, modern laboratory based attosecond light sources allow the measurement of real-time electron dynamics in isolated molecules. ${ }^{20}$

These experiments, however, are themselves inherently not species specific, i.e., all molecules within the interaction region will be probed. Therefore, the combination of LD with XFEL and attosecond experiments requires a pure molecular sample in the gas-phase. Furthermore, to be compatible with central facility light-sources, the laser-desorption source needs to be translatable in three axes to adjust the molecular beam to the fixed XFEL beam. Additionally, the continuous measurement time should be as long as possible and the sample should be quickly exchangeable. 
Here, we detail the characterization and optimization of our novel LDMB source design constructed to be compatible with central facilities, such as XFELs or attoscience centers. Using the dipeptide Ac-Phe-Cys- $\mathrm{NH}_{2}$ as a prototypical labile biological molecule, which has first been laser desorbed and studied by the Rijs group, ${ }^{21,22}$ we characterize the created beam using strong-field ionization with a femtosecond laser pulse. This allows us to monitor all species present in the interaction region, including the carrier gas of the supersonic expansion. We show the optimization of experimental parameters to reduce fragmentation, to improve cooling of desorbed molecules, and, thereby, to maximize the phase-space density of intact parent molecules in the interaction region. The created molecular beams are well suited to further manipulation and purification, e.g., using electrostatic deflection techniques, ${ }^{23}$ an important step towards recording temporally and spatially resolved nuclear and electronic dynamics of isolated biomolecules.

\section{EXPERIMENTAL SETUP}

The mechanical design and construction of this LD source are based on compatibility with large-scale facility-based photon sources. The laser-desorption source consists of a single central mechanical unit containing all necessary parts (molecular beam valve, sample bar with motors, and desorption laser optics). It is mounted on a three-axis manipulator on a single flange for independent motion in the source chamber, which is pumped with a turbo molecular pump (Pfeiffer Vacuum HiPace 700P) to typical operating pressures around $10^{-5}$ mbar. It contains a cantilever piezo valve ${ }^{24}$ operated at 6 bar backing pressure of argon. The valve has a $300 \mu \mathrm{m}$ orifice, followed by a conical opening of $4 \mathrm{~mm}$ length and $40^{\circ}$ opening angle. Conical nozzle shapes are well known to produce molecular beams with more efficient translational cooling and greater directionality, and hence densities, than simple pinhole sources. ${ }^{25}$ A graphite (Poco EDM-1) sample bar ( $80 \mathrm{~mm}$ long, sample channel width $1.2 \mathrm{~mm}$ ) is placed approximately $200 \mu \mathrm{m}$ in front of the valve, see Fig. 1. The sample bar height ( $y$ axis) can be translated using an in-vacuum two-phase stepper motor (Owis SM.255.V6). To replenish the molecular sample, the sample bar can be moved along the $x$-direction using an in-vacuum linear piezo-stage (SmarAct SLC-24120-S-HV), typically operated at $0.02 \mathrm{~mm} / \mathrm{s}$. This results in measurement times of around 70 min per sample bar. For longer measurement periods, the sample bar can be quickly exchanged with a load-lock system, pumped by a Pfeiffer Vacuum HiCube 80 Eco pumpstand (typical turn-around time is $10 \mathrm{~min}$ ). The entire molecule source (valve, sample-bar holder with motors, and desorption-laser optics) is placed on a three-axis manipulator and can furthermore be adjusted for the tip and tilt angle, allowing independent five-axis motion of the device within the vacuum chamber, as required for experiments at XFEL facilities. It is generally useful for the operation of the source in molecular-beam setups where accurate alignment of the source is crucial, e.g., multi-skimmer setups or electrostatic manipulation devices. ${ }^{23,26}$ Detailed drawings of the source and individual components are given in the supplementary material.
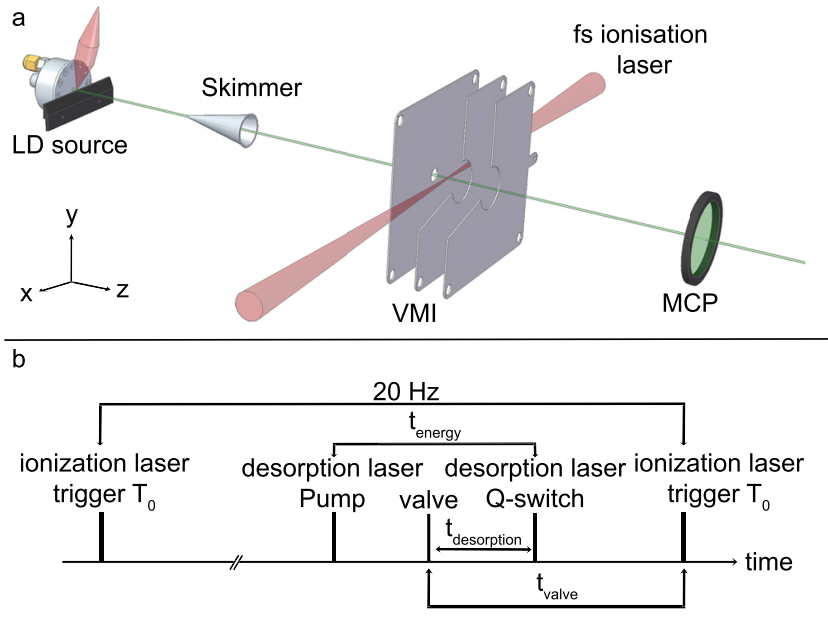

FIG. 1. (a) Experimental setup for characterizing a laser-desorption source. The source chamber contains the laser-desorption source and is separated from the detection chamber by a $2 \mathrm{~mm}$ conical skimmer. The detection chamber houses a velocity-map imaging setup and microchannel plate detector. (b) Experimental timing diagram. The master trigger is given by the ionization laser, a kHz-repetition-rate femtosecond-pulse laser system that cannot trivially be externally triggered, and the molecular beam valve is triggered relative to this with delay $t_{\text {valve }}$. The desorption laser trigger is defined relative to the valve with delay $t_{\text {desorption, }}$, while the energy of the desorption laser can be changed by modifying the Q-switch timing $t_{\text {energy }}$.

Molecules on the sample bar (see below for sample preparation procedures) are desorbed by pulses from a fiber-coupled, diode-pumped Nd:YAG laser at $1064 \mathrm{~nm}$ (Innolas Spitlight Compact DPSS10), operating at $20 \mathrm{~Hz}$ with a pulse duration (full width at half maximum) of 9 ns and pulse energy up to $0.8 \mathrm{~mJ}$. This is coupled into a multimode fiber (CeramOptec WF 400/440P) with core diameter $400 \mu \mathrm{m}$ and numerical aperture of 0.22 . The fiber is coupled into the vacuum chamber with a custom-made Swagelock connection and polytetrafluoroethylene (PTFE) plug. ${ }^{27}$ Inside the chamber, the fiber is out-coupled with a custom-made vacuum compatible fiber collimator, and the laser beam is focused to a spot size of approximately $0.6 \mathrm{~mm}$ on the sample bar. Custom mounting of the collimator allows variation of the laser spot size on the sample, as well as translation of the laser beam along the $x$ and $z$ axes, and tilting in the $y z$ plane.

Following desorption, molecules are picked up by the supersonic argon jet from the valve and rapidly cooled down. The resulting molecular beam is skimmed with a $2 \mathrm{~mm}$ diameter skimmer (Beam Dynamics, Inc., Model 50.8), located approximately $5 \mathrm{~cm}$ downstream of the valve. Following the skimmer, the molecular beam enters the differentially pumped (Pfeiffer Vacuum HiPace 2300) detection chamber, maintained at pressures around $3 \times 10^{-7}$ mbar. The detection chamber contains a velocity-map imaging (VMI) setup with a classic Eppink and Parker 3-plate design. ${ }^{28}$ The distance from the molecular beam valve to the interaction point is around $45 \mathrm{~cm}$. For the results presented here, the VMI setup was operated as a time-of-flight mass spectrometer, with typical mass resolution $m / \Delta m \approx 100$.

The molecular beam is probed via strong-field ionization using a femtosecond Ti:Sapphire laser system (Spectra Physics Spitfire Ace) with a central wavelength of $800 \mathrm{~nm}$, a pulse duration of $40 \mathrm{fs}$, and pulse energies up to $300 \mu \mathrm{J}$. It is focused 
into the vacuum chamber with a $f=800 \mathrm{~mm}$ lens to a spot size (FWHM) of $80 \mu \mathrm{m}$ in the interaction region between the VMI electrodes.

The timing scheme for our experimental setup is shown in Fig. 1. The femtosecond ionization laser is used as the master trigger in the experiment. Its native repetition rate of $1 \mathrm{kHz}$ is electronically reduced to provide a trigger signal at $20 \mathrm{~Hz}$. The molecular beam valve trigger is defined, relative to this fs-laser trigger, by the delay $t_{\mathrm{valve}}$. This delay defines which (longitudinal) part of the molecular beam is probed by the ionization laser. The desorption laser is now triggered relative to the valve trigger and defined by the delay $t_{\text {desorption, }}$ which controls where within the gas pulse the desorbed molecules are placed. This setup enables us to change $t_{\text {valve }}$ without changing $t_{\text {desorption. }}$. We note that the timing values given should be seen as relative, not absolute values, as they are susceptible to electronic delays within the valve and laser controls used.

The velocity of the molecular beam is measured by recording the temporal profile of the beam, i.e., scanning $t_{\text {valve }}$, for different longitudinal positions of the valve, i.e., its distance from the first skimmer. We then evaluate the beam velocity from the temporal shift in the peak of the parent ion for different valve positions to be approximately $670 \mathrm{~m} / \mathrm{s}$.

The dipeptide Ac-Phe-Cys- $\mathrm{NH}_{2}$ (APCN, 95\% purity, antibodies-online $\mathrm{GmbH}$ ) is used in this study without further purification. The sample powder is mixed with graphite powder (0.44:1 by weight) and ground with a pestle and mortar to a fine powder. The top surface of the graphite sample bar is roughened with sand paper and pushed into the prepared sample mixture. Gentle force is used to ensure that the mixture sticks to the sample bar and an even sample layer is formed.

\section{RESULTS AND DISCUSSION}

\section{A. Molecular fragmentation}

A measured time-of-flight mass spectrum of laser desorbed and strong-field ionized APCN is shown in Fig. 2. It shows clear signals from parent ions (1 in Fig. 2), fragment ions (2-6), and the parent dimer (7). Furthermore, we observe several peaks from carbon and carbon clusters consisting of up to 11 atoms (highlighted with asterisks in Fig. 2). These are present due to their direct desorption from the graphite matrix material within which the sample is embedded, as well as due to the formation of higher-order carbon clusters within the desorption plasma created by the laser pulse. ${ }^{29}$ The molecular fragments originating from the APCN sample identified in the spectrum are shown in Fig. 2. Strong-field ionization is a nonspecies-selective method and thus allows the identification of all species present within the molecular beam. This approach, therefore, allows us to optimize the yield and fraction of intact parent molecules contained within the molecular beam. For further analysis of the contributing parameters for laser desorption, we identify four characteristic fragments; the APCN parent ion $(\mathrm{m} / \mathrm{z}=309$, peak labeled 1$)$, the $\mathrm{C}_{10} \mathrm{H}_{12} \mathrm{NO}$ fragment ion $(m / z=162$, peak labeled 3$)$, the argon ion peak $(\mathrm{m} / \mathrm{z}=40)$, and the carbon peak $(\mathrm{m} / \mathrm{z}=12)$. The particular molecular fragment (3) is chosen as it provides the largestintensity clean signal, i.e., it does not overlap with a carbon cluster fragment.

In order to evaluate the effect of the femtosecond ionization laser on the observed molecular fragmentation, we scan the laser pulse energy between $60 \mu \mathrm{J}$ and $220 \mu \mathrm{J}$. The observed integrated ion intensities for the 4 characteristic peaks are shown in Fig. 3(b). The solid lines are a power-law fit of the form $A \times x^{n}+c$. Additionally, in panel (a), we plot the ratio of observed parent ions to the selected fragment ions for the probed intensity region. This nearly constant ratio indicates that the strong-field ionization process has little influence on the fragmentation of the parent ion. This is in agreement with previous studies which indicated that the fragmentation of complex molecules in intense laser fields is very sensitive to the laser pulse duration, but not to the total energy of the pulse. ${ }^{30}$ Therefore, increasing the ionization laser intensities leads to larger ion signals but has little effect on the fragmentation patterns observed. Hence using strong-field ionization with ultrashort laser pulses is a powerful tool for the full characterization of molecular beams containing complex molecules and fragments thereof.

To elucidate the effect of the high-power desorption laser on the molecular sample on the graphite sample bar, we record mass spectra for various desorption laser energies in the range $\sim 350-800 \mu \mathrm{J}$. In Fig. 3(d), we plot the recorded integrated
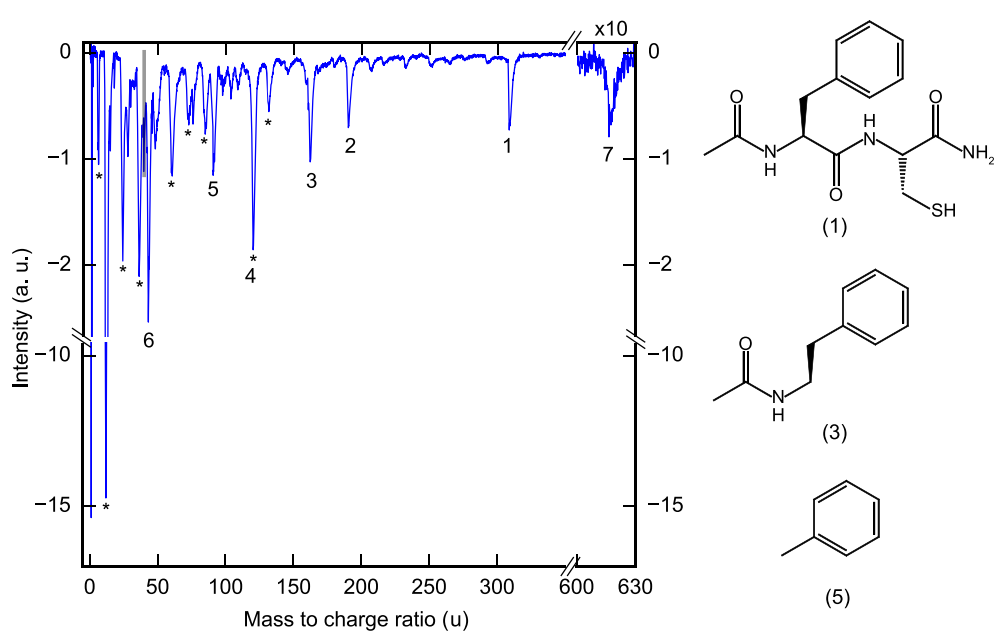

(5)

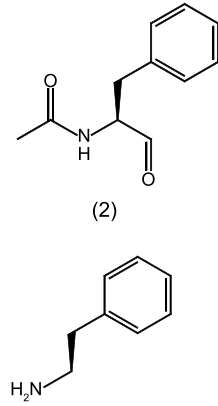

(4)

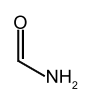

(6)
FIG. 2. Time-of-flight mass spectrum of laser desorbed APCN following strong-field ionization. Peaks resulting from carbon or carbon clusters are labeled with $*$. 1 corresponds to the parent ion, and 2-6 correspond to molecular fragments. A small signal from the APCN dimer is also observed, 7. Gray shading marks the peak from argon carrier gas, which appears much smaller than molecular fragments due to the much higher ionization potential. 

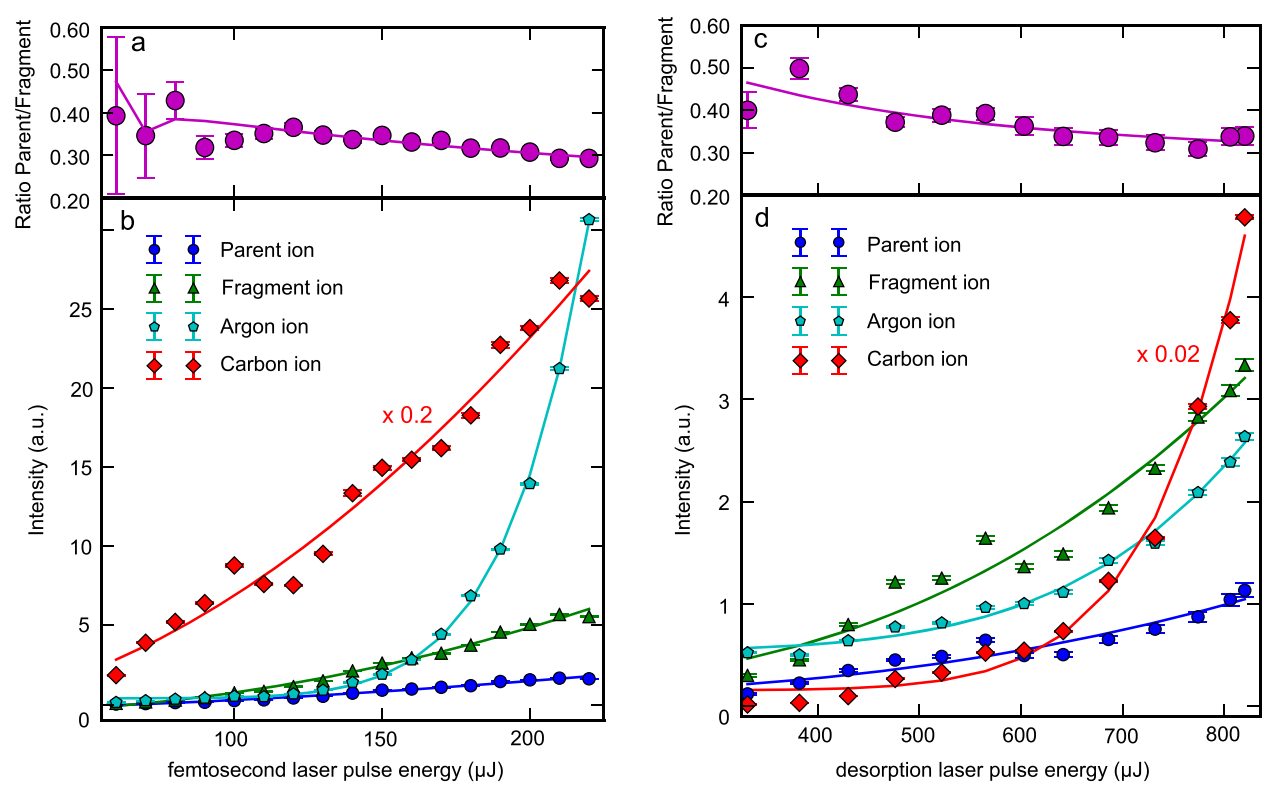

FIG. 3. Measured ion intensity for parent APCN, the characteristic fragment at $m / z=162$, carbon and argon as a function of the ionization laser pulse energy [(a) and (b)] and desorption laser pulse energy [(c) and (d)]. Solid lines are power law fits to the data. The top panels [(a) and (c)] show the ratio of parent to fragment ions observed. Throughout the manuscript, shown error bars correspond to 1 standard error (std. err.).

ion intensities for the four characteristic masses as a function of desorption laser energy, with solid lines corresponding to a power law fit to the data. As can be seen from these data, all intensities increase with increasing laser energy. This includes, somewhat unintuitively, the argon seed gas signal observed in the interaction region, which will be discussed later on. The carbon signal shows the steepest dependence on desorption laser pulse energy, which is consistent with the formation of isolated carbon atoms and clusters within a laserinduced plasma. ${ }^{29}$ Figure 3(c) shows the ratio of the parent to fragment signal. A decrease in the parent-to-fragment ratio is observed as the desorption energy is increased. Thus the desorption process can cause fragmentation of the sample, and the highest fraction of the parent ion within the molecular beam is obtained at the lowest desorption energies, albeit at the expense of density within the beam. We furthermore note that the actual fractional yield of parent ions within the molecular beam is significantly smaller than the numerical values shown in Fig. 3 since these only take into account a single characteristic fragment. Additionally, any charged fragments produced during the desorption process will not arrive in the detection region due to the static fields applied to the time-of-flight electrodes. All studies below were conducted with around 670 $\mu \mathrm{J}$ desorption laser pulse energy and $140 \mu \mathrm{J}$ ionization laser power.

\section{B. Molecular beam properties}

To probe the longitudinal (or temporal) profile of the produced molecular beam, we scan the timing between the valve trigger and the ionization laser, $t_{\mathrm{valve}}$, see Fig. 1, probing different portions of the molecular beam. A typical temporal profile is shown in Fig. 4(a) for the pure argon beam emerging from the valve with (purple) and without (turquoise) the graphite sample bar in place. Without the sample bar, we observe a single sharp peak with a full width at half maximum of $\sim 75 \mu$ s (corresponding to a speed ratio of $\sim 10$ ) and a small shoulder at longer times due to the rebounce of the piezo within the valve. ${ }^{24}$ When the sample bar is placed in front of the valve, as shown in the inset of Fig. 4, the argon gas flow is significantly disturbed. The overall gas-pulse is significantly broader, the main peak intensity is decreased by a factor of $\sim 3$, and more intensity is observed at later times. We attribute these observations to the disturbance of the argon flow by the sample bar and possible turbulences in the flow-field within the dead volume behind the sample bar.

By changing the relative timing of the desorption laser and valve trigger ( $t_{\text {desorption }}$ in Fig. 1$)$, we can now place the plume of desorbed molecules at different positions within the argon beam. Changing this relative timing has significant effects on the observed intensities of argon, parent, and fragment ions alike, as shown in Fig. 4(b). We show temporal molecular beam profiles for argon (diamond markers), fragment 3 (triangles), and parent ions (circles) at two different

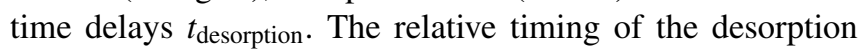
laser and molecular beam valve significantly affects the intensity of fragment and parent ions and the ratio between the two. We attribute this effect to changes in the cooling efficiency as the hot desorbed molecules are placed within the argon expansion at different times. An efficient cooling process is required to quench the excess energy of the desorbed molecules and prevent further fragmentation. Comparing the relative integrated intensities of parent and fragment signals at the two timings shown in Fig. 4(b), we observe that the combined intensity is approximately identical at the two time points; however, the ratio between the two differs significantly. This suggests that while approximately the same density of molecules, including fragments, is present within the initially desorbed plume, the less efficient cooling in the less-dense front of the gas pulse at the delay of $40 \mu$ s leads to significant fragmentation occurring before or during the argon gas pulse, i.e., cooling occurs too late and fragmentation has already taken place.

Further to its influence on the molecular signals, the timing of the desorption laser clearly has an effect on the observed argon signal. An increase in the argon signal is observed at 


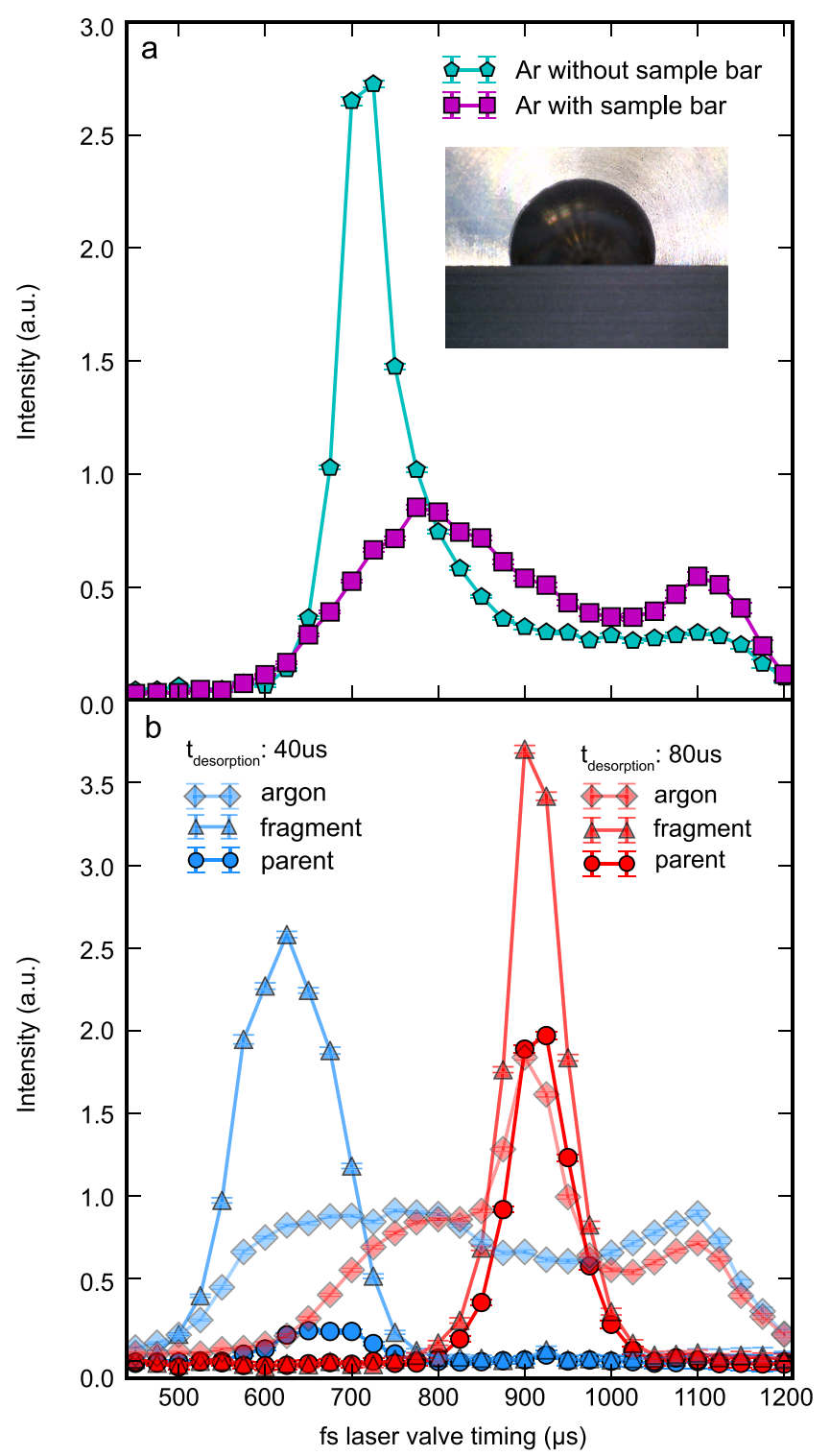

FIG. 4. Integrated ion intensity as a function of valve delay $t_{\text {valve }}$, i.e., temporal (longitudinal) profiles of the molecular beam. (a) Argon profile with (purple squares) and without (turquoise pentagons) the sample bar in front of the valve. The inset shows a photograph of the sample bar in front of the valve at the optimized position; see the text for details. (b) Temporal profile for the APCN parent, the characteristic fragment, and argon for two different desorption laser delays, $t_{\text {desorption }}$.

the timing where desorbed molecules (parent or fragments) are present within the beam. We suspect the observed increase in the argon signal is not due to more argon arriving at the detector, but due to signals of molecular fragments or carbon clusters overlapping with the argon mass at $40 \mathrm{u}$ in a very crowded spectrum shown in Fig. 2.

The dependence of the observed fragmentation on the relative position in the gas pulse is, furthermore, evident when comparing mass spectra recorded at different $t_{\text {desorption, }}$ as shown in Fig. 5. These spectra have been recorded with $t_{\mathrm{valve}}$ optimized for the parent ion signal and are plotted normalized with respect to the observed parent intensity. This shows that all molecular fragments are significantly more abundant at $t_{\text {desorption }}=40 \mu \mathrm{s}$, indicating a much higher internal temperature of the desorbed molecules, due

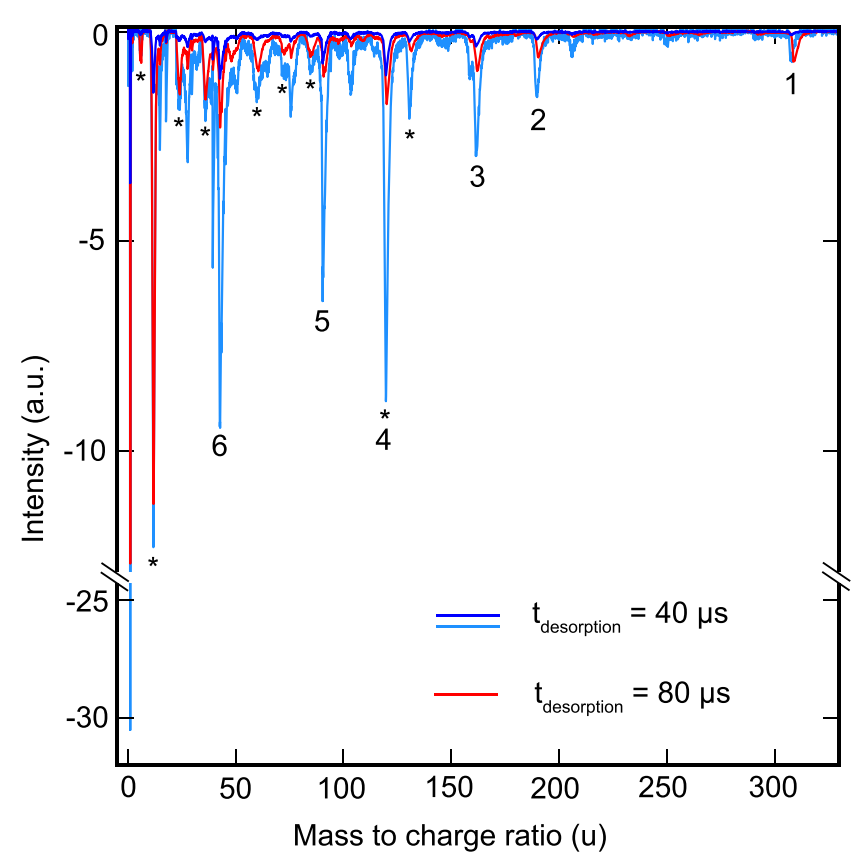

FIG. 5. Mass spectrum for the two different desorption laser timings shown in Fig. 4(b); red and dark blue are the absolute intensities measured and the light blue trace has been scaled to the parent ion intensity of the red $\left(t_{\text {desorption }}=80\right.$ $\mu s)$ trace. A significant increase in the fragment ion yield is observed for $t_{\text {desorption }}=40 \mu \mathrm{s}$.

to the less efficient cooling, and correspondingly increased fragmentation.

To identify the optimum placement of the desorbed plume within the gas pulse, we have repeated these measurements for several delay points, shown in Fig. 6, where the curve with a higher intensity always corresponds to the fragment and the curve with a lower intensity corresponds to the parent ion. For better comparison, the parent intensity for each $t_{\text {desorption }}$, as well as the ratio of the parent to the characteristic fragment, is shown in Fig. 7.

For our setup and under the given experimental conditions, we identify a delay of $80 \mu \mathrm{s}$ as providing the highest total intensity of the parent signal, as well as the best parent-fragment ratio. This ratio is very sensitive to the relative timings, and changes of $10 \mu$ s can change this ratio by a factor of $\sim 2$. This is due to the gas pulse directly after the valve being significantly shorter (opening time of the piezo is around $25 \mu \mathrm{s}$ ) than in the detection region located $\sim 0.5 \mathrm{~m}$ downstream where the measurements were taken. This is also reflected by the steep falloff of signals for later desorption laser timings.

As shown above, the sample bar has a large effect on the supersonic expansion and hence the produced molecular beam. To investigate this further, we have taken data for different heights of the sample bar, shown in Fig. 8. Here, we recorded the intensity of argon, carbon, fragment, and parent ions for different heights of the sample bar, as well as the ratio of detected parent to fragment ions. We note that all these data were taken at identical timings of $t_{\mathrm{valve}}=-900 \mu \mathrm{s}$ and $t_{\text {desorption }}=80 \mu \mathrm{s}$ and that the height of the sample bar does change the desorption laser focusing conditions (since the focusing lens is fixed relative to the valve). 


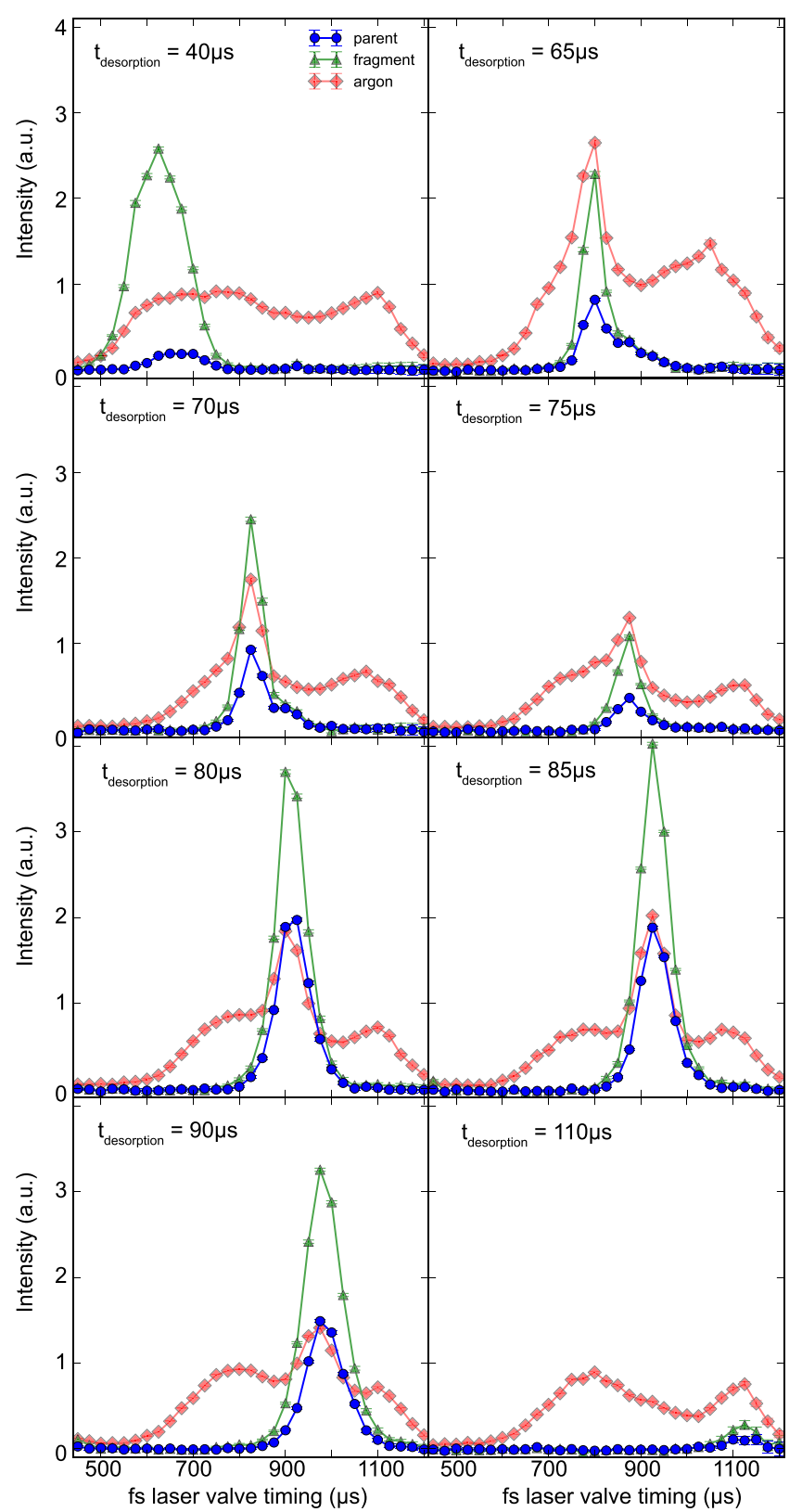

FIG. 6. Longitudinal (temporal) profiles of the molecular beam for different

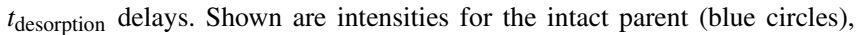
fragment (green triangles), and argon (red squares) ions.

It is evident from these data that the sample bar height changes not only the peak intensity but also the parent to fragment ratio, as shown in Fig. 8(a). The observed intensities are very sensitive to the height of the sample bar, with parent, fragment, and carbon ions showing maxima at different positions. This sensitivity was used in all previous measurements to fix the height for each new sample bar measured; it was optimized prior to taking data to obtain maximum signals from the parent ion. However, we note that due to the preparation method, slight differences in height can persist even across a single sample bar and affect the measurement, especially the comparability between data sets. This could be the reason for the large deviation of the data shown for $75 \mu$ s in Fig. 7. This sensitivity can be explained by a number of contributing factors: (i) the efficiency with which desorbed molecules are picked up and carried by the argon beam. Assuming that the

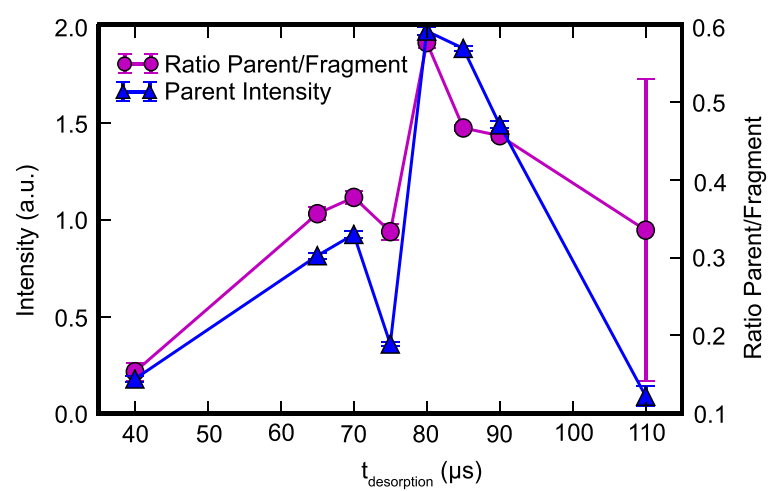

FIG. 7. Dependence of the parent ion intensity and the parent to fragment ratio on the desorption laser timing $t_{\text {desorption }}$; error bars correspond to 1 standard error. Optimum conditions are observed for $t_{\text {desorption }}=80 \mu \mathrm{s}$. The data at $75 \mu$ s show a significantly lower intensity than expected, which could be due to irregularities in sample bar preparation; see the text for details.

slight differences in mass and size between parent and fragments are negligible, given the very large number of collisions with the carrier gas, this should be comparable for all species within the beam, i.e., a lower pressure of argon should affect all species to a comparable extent. (ii) The degree to which the argon expansion is disturbed by the presence of the sample bar. In Fig. 4(a), we have shown that the sample bar influences the molecular beam speed and distribution; at the fixed $t_{\text {valve }}$ conditions used here, this will change the observed intensities. It has furthermore been shown already that the sample bar changes

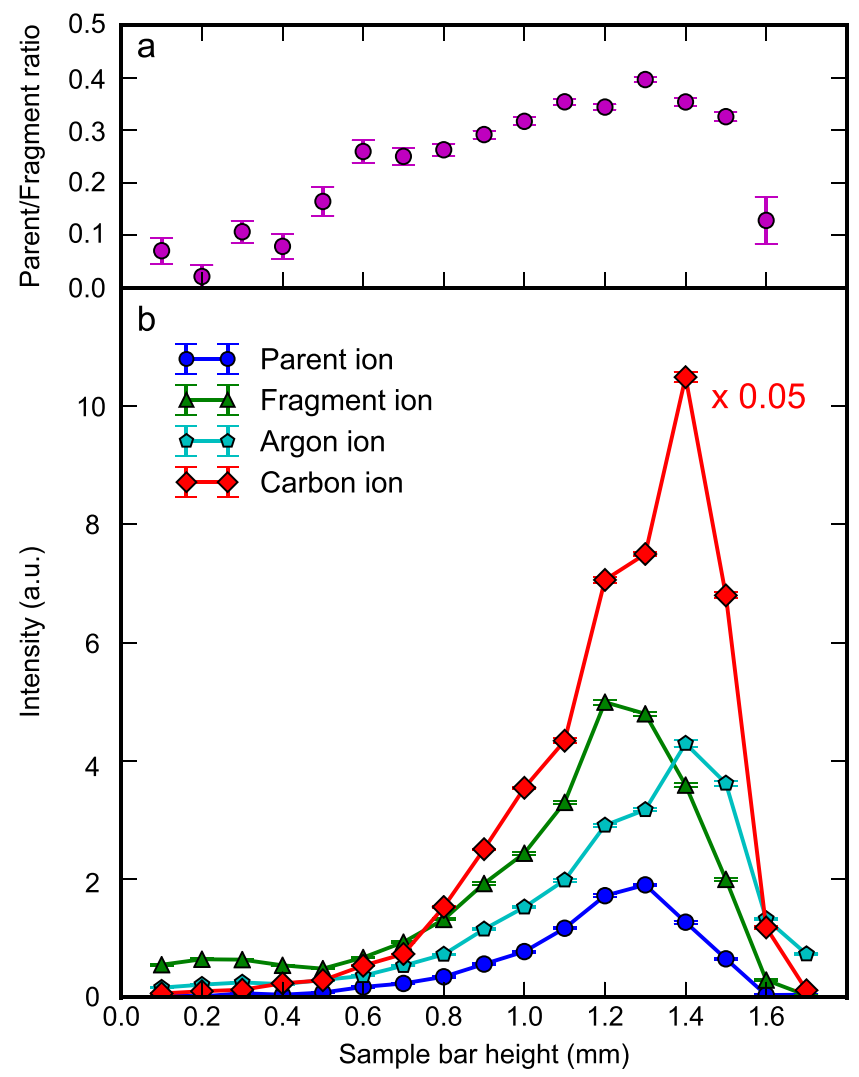

FIG. 8. Measured ion intensity for the parent APCN, the characteristic fragment at $m / z=162$, carbon and argon as a function of the sample bar height. The top panel shows the ratio of parent to fragment ions observed. 
the directionality of the molecular beam. ${ }^{31}$ (iii) Changes in the cooling efficiency of desorbed species lead to differences in the parent to fragment ratio in the beam. (iv) The spot size of the desorption laser on the sample bar changes with different sample bar heights, and thus the intensity of the laser and the number of molecules interacting with the laser are influenced. From the collected data, we cannot comment on the relative importance of these different mechanisms; however, since (i) and (ii) should influence the parent and fragment molecules nearly identically, the observed changes in the parent to fragment ratio indicate a dependence of the cooling efficiency on the sample-bar height. The cooling efficiency should be best within the densest part of the molecular beam, which is on the axis of the $300 \mu \mathrm{m}$ nozzle orifice. Therefore, a sample bar height just below this position, i.e., covering slightly less than half the nozzle opening, should lead to the densest plume of desorbed molecules being entrained in the densest part of the molecular beam. This simple consideration is consistent with our observations of the maximum parent signal and parent to fragment ratio occurring at the position labeled $1.3 \mathrm{~mm}$. While we cannot disentangle all the different effects of changing the sample bar height, it is clear that this, and the associated influence on the supersonic expansion and molecular beam properties, is a crucial parameter for laser desorption entrainment of molecules into supersonic expansions. This could be further investigated either by measuring the gas flow from the nozzle directly, for example, through direct visualization of gas densities, ${ }^{25,32}$ or by measuring spatial argon profiles through strong-field-ionization mass spectrometry at various distances from the nozzle.

Despite the wide use of laser desorption sources, very few studies have looked into the fundamental underlying processes. Furthermore, the vastly different source designs in use, e.g., different desorption laser wavelengths, intensities, pulse durations, different models of supersonic valves, etc., make comparison to previous studies difficult. While we believe this is the first study of laser desorption using strong-field ionization, previous experiments have utilized electron impact ionization and have similarly observed a large amount of neutral fragments produced by the desorption processes. ${ }^{1}$ Our finding that the molecular packet of desorbed molecules is much shorter than the envelope of the seeding-gas pulse is also consistent with previous measurements. ${ }^{31}$ Finally, we point out that the use of a fiber-coupled desorption laser has been demonstrated before, ${ }^{33}$ albeit without refocusing inside the vacuum chamber that we have introduced here for greater control.

\section{CONCLUSION}

We have presented a novel laser-desorption setup designed for use in advanced imaging experiments of ultrafast molecular dynamics, and we have carefully characterized and optimized the laser-desorption and molecular-beam-entrainment conditions. The setup consists of a single central mechanical unit containing all necessary parts (molecular beam valve, sample bar with motors, and desorption laser optics) that is mounted on an $X Y Z$ manipulator on a single flange for independent motion. Furthermore, we have presented a detailed characterization of our new laser-desorption source as well as molecular beams produced using laser desorption in general. By utilizing strong-field ionization, we were able to probe all species contained within the beam. Under normal operating conditions, we found that the molecular beam contains, in addition to parent molecules, significant amounts of molecular fragments, as well as carbon clusters from the desorption process. We investigated the role of the desorption laser fluence, the relative timing of valve opening and desorption laser, the sample bar height, and which part of the molecular packet is probed. While increased desorption laser fluence leads to more molecules contained within the molecular beam, it was found to induce fragmentation of the sample and leads to enhanced contamination of the beam with carbon and its clusters. The placement of the desorbed plume of molecules within the gas pulse from the supersonic expansion has a profound effect on the cooling efficiency and thus the fragmentation observed. The best timing was found to be approximately in the center of the gas pulse and is quite sensitive compared with the gas pulse duration in the detection region. The relative height of the sample bar in front of the valve orifice significantly affects the molecular beam expansion conditions, and hence the intensity of observed signals, as well as the parent to fragment ratio. However, finding the optimum position for the sample bar height is difficult due to the number of competing effects taking place, and every sample bar being unique. Furthermore, parameters might be dependent on the employed molecularbeam nozzle, and our exact findings are specific to the used conical nozzle shape.

From our detailed investigation, we found that the optimal settings for building a laser-desorption source very much depend on the planned experimental scheme. While some parameters, such as the relative timing of desorption laser and the molecular beam valve, should always be optimized as shown here, other parameters are not critical. For example, the pulse energy of the desorption laser should be chosen according to the application. For techniques that are only sensitive to the intact parent molecule signal, like resonance-enhanced multiphoton ionization, the pulse energy of the desorption laser should be kept high because this increases the number density of parent molecules in the interaction region. But for nonspecies specific techniques, such as X-ray diffraction, the pulse energy should be reduced to minimize the contamination with fragments and carbon. However, even at the lowest desorption energy used here, we still observe a significant amount of molecular fragments and carbon clusters in the beam. While the former originate to some extent from the strong-fieldionization probing, carbon and carbon clusters are certainly in the beam due to the desorption process. In order to produce a pure beam of intact parent molecules in the gas-phase, one can consider coupling a laser-desorption source with other species separation techniques for neutral molecules, such as electrostatic deflection or alternating gradient focusing, ${ }^{23,34}$ and such experiments are currently underway in our laboratory.

\section{SUPPLEMENTARY MATERIAL}

See supplementary material for detailed 3D drawings of the laser desorption source and individual components. 


\section{ACKNOWLEDGMENTS}

We thank Anouk Rijs for helpful discussions regarding the design of our new source, Horst Zink and the DESY FS electronics workshop for expert support with the setup, and Ortwin Hellmig and Andreas Bick for help with the fiber vacuum feedthrough.

In addition to DESY, this work has been supported by the European Research Council under the European Union's Seventh Framework Programme (No. FP7/2007-2013) through the Consolidator Grant COMOTION (No. ERC-614507-Küpper), by the excellence cluster "The Hamburg Center for Ultrafast Imaging - Structure, Dynamics and Control of Matter at the Atomic Scale" of the Deutsche Forschungsgemeinschaft (CUI, DFG-EXC1074), and by the Helmholtz Gemeinschaft through the "Impuls- und Vernetzungsfond." We gratefully acknowledge a Kekulé Mobility Fellowship by the Fonds der Chemischen Industrie (FCI) for Nicole Teschmit.

${ }^{1}$ F. J. Vastola and A. J. Pirone, "Ionization of organic solids by laser irradiation," Adv. Mass Spectrom. 4, 107 (1968).

${ }^{2}$ A. M. Rijs and J. Oomens, "IR spectroscopic techniques to study isolated biomolecules," in Gas-Phase IR Spectroscopy and Structure of Biological Molecules, edited by A. M. Rijs and J. Oomens (Springer Verlag, 2015), Chap. 1, pp. 1-42.

${ }^{3}$ R. Tembreull and D. M. Lubman, "Resonant two-photon ionization of small peptides using pulsed laser desorption in supersonic beam mass spectrometry," Anal. Chem. 59, 1003-1006 (1987).

${ }^{4}$ G. Meijer, M. S. de Vries, H. E. Hunziker, and H. R. Wendt, "Laser desorption jet-cooling of organic molecules-Cooling characteristics and detection sensitivity," Appl. Phys. B 51, 395-403 (1990).

${ }^{5}$ E. Nir, K. Kleinermanns, and M. S. de Vries, "Pairing of isolated nucleicacid bases in the absence of the DNA backbone," Nature 408, 949-951 (2000).

${ }^{6}$ M. S. de Vries and P. Hobza, "Gas-phase spectroscopy of biomolecular building blocks," Annu. Rev. Phys. Chem. 58, 585-612 (2007).

${ }^{7}$ S.-i. Ishiuchi, K. Yamada, H. Oba, H. Wako, and M. Fujii, "Gas phase ultraviolet and infrared spectroscopy on a partial peptide of $\beta_{2}$-adrenoceptor SIVSF- $\mathrm{NH}_{2}$ by a laser desorption supersonic jet technique," Phys. Chem. Chem. Phys. 18, 23277-23284 (2016).

${ }^{8}$ J. M. Bakker, C. Plützer, I. Hünig, T. Häber, I. Compagnon, G. von Helden, G. Meijer, and K. Kleinermanns, "Folding structures of isolated peptides as revealed by gas-phase mid-infrared spectroscopy," Chem. Phys. Chem. 6, 120-128 (2005).

${ }^{9}$ J. M. Bakker, L. M. Aleese, G. Meijer, and G. von Helden, "Fingerprint IR spectroscopy to probe amino acid conformations in the gas phase," Phys. Rev. Lett. 91, 203003 (2003).

${ }^{10}$ M. R. Ligare, A. M. Rijs, G. Berden, M. Kabeláč, D. Nachtigallova, J. Oomens, and M. S. de Vries, "Resonant infrared multiple photon dissociation spectroscopy of anionic nucleotide monophosphate clusters," J. Phys. Chem. B 119, 7894-7901 (2015).

${ }^{11}$ J. Zhang, L. Pei, and W. Kong, "Zero kinetic energy photoelectron spectroscopy of tetracene using laser desorption for vaporization," J. Chem. Phys. 128, 104301 (2008).

${ }^{12}$ A. Barty, J. Küpper, and H. N. Chapman, "Molecular imaging using x-ray free-electron lasers," Annu. Rev. Phys. Chem. 64, 415-435 (2013).

${ }^{13}$ R. Neutze, R. Wouts, D. van der Spoel, E. Weckert, and J. Hajdu, "Potential for biomolecular imaging with femtosecond $\mathrm{x}$-ray pulses, "Nature 406, 752757 (2000).

${ }^{14}$ B. Ziaja, H. N. Chapman, R. Fäustlin, S. Hau-Riege, Z. Jurek, A. V. Martin, S. Toleikis, F. Wang, E. Weckert, and R. Santra, "Limitations of coherent diffractive imaging of single objects due to their damage by intense $\mathrm{x}$-ray radiation," New J. Phys. 14, 115015 (2012).

${ }^{15}$ U. Lorenz, N. M. Kabachnik, E. Weckert, and I. A. Vartanyants, "Impact of ultrafast electronic damage in single-particle x-ray imaging experiments," Phys. Rev. E 86, 051911 (2012); e-print arXiv:1206.6960 [physics].

${ }^{16}$ K. Nass, L. Foucar, T. R. M. Barends, E. Hartmann, S. Botha, R. L. Shoeman, R. B. Doak, R. Alonso-Mori, A. Aquila, S. Bajt, A. Barty, R. Bean, K. R. Beyerlein, M. Bublitz, N. Drachmann, J. Gregersen, H. O. Jönsson,
W. Kabsch, S. Kassemeyer, J. E. Koglin, M. Krumrey, D. Mattle, M. Messerschmidt, P. Nissen, L. Reinhard, O. Sitsel, D. Sokaras, G. J. Williams, S. Hau-Riege, N. Timneanu, C. Caleman, H. N. Chapman, S. Boutet, and I. Schlichting, "Indications of radiation damage in ferredoxin microcrystals using high-intensity X-FEL beams," J. Synchrotron Radiat. 22, 225-238 (2015).

${ }^{17}$ J. Küpper, S. Stern, L. Holmegaard, F. Filsinger, A. Rouzée, A. Rudenko, P. Johnsson, A. V. Martin, M. Adolph, A. Aquila, S. Bajt, A. Barty, C. Bostedt, J. Bozek, C. Caleman, R. Coffee, N. Coppola, T. Delmas, S. Epp, B. Erk, L. Foucar, T. Gorkhover, L. Gumprecht, A. Hartmann, R. Hartmann, G. Hauser, P. Holl, A. Hömke, N. Kimmel, F. Krasniqi, K.-U. Kühnel, J. Maurer, M. Messerschmidt, R. Moshammer, C. Reich, B. Rudek, R. Santra, I. Schlichting, C. Schmidt, S. Schorb, J. Schulz, H. Soltau, J. C. H. Spence, D. Starodub, L. Strüder, J. Thøgersen, M. J. J. Vrakking, G. Weidenspointner, T. A. White, C. Wunderer, G. Meijer, J. Ullrich, H. Stapelfeldt, D. Rolles, and H. N. Chapman, "X-ray diffraction from isolated and strongly aligned gas-phase molecules with a free-electron laser," Phys. Rev. Lett. 112, 083002 (2014); e-print arXiv:1307.4577 [physics].

${ }^{18}$ S. Stern, L. Holmegaard, F. Filsinger, A. Rouzée, A. Rudenko, P. Johnsson, A. V. Martin, A. Barty, C. Bostedt, J. D. Bozek, R. N. Coffee, S. Epp, B. Erk, L. Foucar, R. Hartmann, N. Kimmel, K.-U. Kühnel, J. Maurer, M. Messerschmidt, B. Rudek, D. G. Starodub, J. Thøgersen, G. Weidenspointner, T. A. White, H. Stapelfeldt, D. Rolles, H. N. Chapman, and J. Küpper, "Toward atomic resolution diffractive imaging of isolated molecules with x-ray freeelectron lasers," Faraday Discuss. 171, 393 (2014); e-print arXiv: 1403.2553 [physics].

${ }^{19}$ J. M. Glownia, A. Natan, J. P. Cryan, R. Hartsock, M. Kozina, M. P. Minitti, S. Nelson, J. Robinson, T. Sato, T. van Driel, G. Welch, C. Weninger, D. Zhi, and P. H. Bucksbaum, "Self-referenced coherent diffraction X-ray movie of angstrom- and femtosecond-scale atomic motion," Phys. Rev. Lett. 117, 153003 (2016); e-print arXiv:1608.03039 [physics].

${ }^{20}$ F. Calegari, D. Ayuso, A. Trabattoni, L. Belshaw, S. De Camillis, S. Anumula, F. Frassetto, L. Poletto, A. Palacios, P. Decleva, J. B. Greenwood, F. Martín, and M. Nisoli, "Ultrafast electron dynamics in phenylalanine initiated by attosecond pulses," Science 346, 336-339 (2014).

${ }^{21}$ B. Yan, S. Jaeqx, W. J. van der Zande, and A. M. Rijs, "A conformationselective IR-UV study of the dipeptides Ac-Phe-Ser-NH $\mathrm{N}_{2}$ and Ac-Phe-Cys$\mathrm{NH}_{2}$ : Probing the $\mathrm{SH} \cdots \mathrm{O}$ and $\mathrm{OH} \cdots$ O hydrogen bond interactions," Phys. Chem. Chem. Phys. 16, 10770-10778 (2014).

${ }^{22}$ M. Alauddin, H. S. Biswal, E. Gloaguen, and M. Mons, "Intra-residue interactions in proteins: Interplay between serine or cysteine side chains and backbone conformations, revealed by laser spectroscopy of isolated model peptides," Phys. Chem. Chem. Phys. 17, 2169-2178 (2015).

${ }^{23}$ Y.-P. Chang, D. A. Horke, S. Trippel, and J. Küpper, "Spatially-controlled complex molecules and their applications," Int. Rev. Phys. Chem. 34, 557590 (2015); e-print arXiv:1505.05632 [physics].

${ }^{24}$ D. Irimia, D. Dobrikov, R. Kortekaas, H. Voet, D. A. van den Ende, W. A. Groen, and M. H. M. Janssen, "A short pulse (7 $\mu$ s FWHM) and high repetition rate $(\mathrm{dc}-5 \mathrm{kHz})$ cantilever piezovalve for pulsed atomic and molecular beams," Rev. Sci. Instrum. 80, 113303 (2009).

${ }^{25} \mathrm{~K}$. Luria, W. Christen, and U. Even, "Generation and propagation of intense supersonic beams,” J. Phys. Chem. A 115, 7362-7367 (2011).

${ }^{26}$ H. L. Bethlem, M. R. Tarbutt, J. Küpper, D. Carty, K. Wohlfart, E. A. Hinds, and G. Meijer, "Alternating gradient focusing and deceleration of polar molecules," J. Phys. B 39, R263-R291 (2006); e-print arXiv:0604020 [physics].

${ }^{27}$ E. R. Abraham and E. A. Cornell, "Teflon feedthrough for coupling optical fibers into ultrahigh vacuum systems," Appl. Opt. 37, 1762-1763 (1998).

${ }^{28}$ A. T. J. B. Eppink and D. H. Parker, "Velocity map imaging of ions and electrons using electrostatic lenses: Application in photoelectron and photofragment ion imaging of molecular oxygen," Rev. Sci. Instrum. 68, 3477-3484 (1997).

${ }^{29}$ E. A. Rohlfing, D. M. Cox, and A. Kaldor, "Production and characterization of supersonic carbon cluster beams," J. Chem. Phys. 81, 3322-3330 (1984).

${ }^{30}$ C. R. Calvert, L. Belshaw, M. J. Duffy, O. Kelly, R. B. King, A. G. Smyth, T. J. Kelly, J. T. Costello, D. J. Timson, W. A. Bryan, T. Kierspel, P. Rice, I. C. E. Turcu, C. M. Cacho, E. Springate, I. D. Williams, and J. B. Greenwood, "LIAD-fs scheme for studies of ultrafast laser interactions with gas phase biomolecules," Phys. Chem. Chem. Phys. 14, 6289-6297 (2012).

${ }^{31}$ P. Arrowsmith, M. S. de Vries, H. E. Hunziker, and H. R. Wendt, "Pulsed laser desorption near a jet orifice: Concentration profiles of entrained perylene vapor," Appl. Phys. B 46, 165-173 (1988). 
${ }^{32}$ D. A. Horke, N. Roth, L. Worbs, and J. Küpper, "Characterizing gas flow from aerosol particle injectors," J. Appl. Phys. 121, 123106 (2017); e-print arXiv:1609.09020 [physics].

${ }^{33}$ F. Piuzzi, I. Dimicoli, M. Mons, B. Tardivel, and Q. C. Zhao, "A simple laser vaporization source for thermally fragile molecules coupled to a supersonic expansion: Application to the spectroscopy of tryptophan," Chem. Phys. Lett. 320, 282-288 (2000).

${ }^{34}$ F. Filsinger, U. Erlekam, G. von Helden, J. Küpper, and G. Meijer, "Selector for structural isomers of neutral molecules," Phys. Rev. Lett. 100, 133003 (2008); e-print arXiv:0802.2795 [physics]. 